\title{
Qualités morpho-physiologiques et évaluation du comportement germinatif des graines du théier des savanes (Lippia multiflora Moldenke)
}

\author{
Hervé Cédessia Kéassemon KONE ${ }^{*}$, Nicaise Tetchi AKEDRIN², Vama Etienne TIA², \\ Fatou BAYOKO ${ }^{1}$ et Lacina Fanlégué COULIBALY ${ }^{1}$
}

\author{
'Département de Biologie Végétale, UFR des Sciences Biologiques, Université Péléfero Gon Coulibaly BP \\ 1328 Korhogo. \\ ${ }^{2}$ Laboratoire d'Amélioration de la Production Agricole, UFR Agroforesterie, Université Jean Lorougnon \\ Guédé, BP 150 Daloa. \\ ${ }^{3}$ Département de Biochimie-génétique, UFR des Sciences Biologiques, Université Péléfero Gon Coulibaly BP \\ 1328 Korhogo. \\ *Auteur correspondant ; E-mail : hervekone2001@gmail.com
}

\section{RESUME}

La domestication de Lippia multiflora, plantes aux multiples vertus en pharmacopée et médecine est un enjeu de taille en Côte d'Ivoire. Cependant, l'insuffisance de semences, due au faible taux de germination des graines, limite l'extension de sa culture. La présente étude avait pour objectif l'extraction, la purification et la caractérisation morpho-physiologique des graines de Lippia multiflora Moldenke (Verbenaceae). Il s'agissait plus spécifiquement de déterminer la pureté spécifique, le nombre de graines par unité de masse, le diamètre moyen et le taux d'humidité ; le taux de germination des graines de L. multiflora. Aussi, il s'est agi d'étudier l'impact du milieu sur le pouvoir germinatif des graines et de faire un suivi post-germination des plants en milieu réel. Après extraction des graines, des mesures physiques et des tests de germination ont permis de déterminer les caractéristiques morpho-physiologiques de celles-ci. L'étude a montré que le lot de graine étudié a une pureté spécifique de $70 \%$, les graines ont un diamètre moyen de $0,34 \pm 0.1 \mathrm{~mm}$, une teneur en eau de $14 \pm 5.4 \%$ et un taux de germination de $42,25 \%$. Les tests de germination après un séjour prolongé dans divers milieux, révèlent qu'un milieu réfrigéré $\left(7^{\circ} \mathrm{C}\right)$ confère une plus longue viabilité aux graines. Le suivi post-germination au champ montre une évolution régulière de la hauteur des plants, passant en moyenne de 2,58 $\mathrm{cm}$ à $8,8 \mathrm{~cm}$ au bout de 3 mois. Sur la même période, le nombre moyen de feuille varie de 4,03 à 21 .

(C) 2020 International Formulae Group. All rights reserved.

Mots clés : Lippia multiflora, graine, caractérisation, germination, suivi post-germination.

\section{Morpho-physiological qualities and evaluation of the germination behavior of seeds of the savannah tea tree (Lippia multiflora Moldenke)}

\begin{abstract}
The domestication of Lippia multiflora, plants with multiple virtues in pharmacopoeia and medicine is a major challenge in Côte d'Ivoire. However, the lack of seed, due to the low germination rate, limits the extension of its cultivation. The purpose of this study was the extraction, purification and morpho-physiological characterization of the seeds of Lippia multiflora Moldenke (Verbenaceae). More specifically, it involved determining the specific
\end{abstract}


purity, the number of seeds per unit mass, the average diameter and the humidity rate; the germination rate of $L$. multiflora seeds. Also, it was a question of studying the impact of the environment on the germination power of seeds and of making a post-germination follow-up of the plants in real environment. After the seeds extraction, physical measurements and germination tests were carried out to determine the morpho-physiological characteristics. Results showed that the seed lot studied has a specific purity of $70 \%$, an average diameter of 0.34 $0.1 \mathrm{~mm}$, a water content of $145.4 \%$ and a germination rate of $42.25 \%$. Germination tests after a prolonged stay in various environments reveal that a refrigerated area $\left(7^{\circ} \mathrm{C}\right)$ confers a longer viability to the seeds. Postgermination monitoring in the field shows a steady increase in plant height from an average of $2.58 \mathrm{~cm}$ to $8.8 \mathrm{~cm}$ after 3 months. Over the same period, the average number of sheets varies from 4.03 to 21 .

(C) 2020 International Formulae Group. All rights reserved.

Keywords: Lippia multiflora, seed, characterization, germination, post-germination monitoring.

\section{INTRODUCTION}

Lippia multtiflora Moldenke est une plante aromatique qui croit spontanément dans les zones de savane de l'Afrique subtropicale. Dans les pays tropicaux en général et en Afrique sub-saharienne en particulier, plus de $80 \%$ de la population rurale continue de recourir aux plantes sauvages pour l'alimentation et les soins de santé primaires (Ambé, 2001). Communément appelée « théier de savane » ou "théier de Gambie », elle est traditionnellement utilisée pour soigner les affections bronchiques, les accès fébriles, la rhinopharyngite, les conjonctivites et les ictères. Cette plante, qui entre dans la composition de certains médicaments traditionnels améliorés africains (Malarial ${ }^{\circledR}$ au Mali, et le Tétra ${ }^{\circledR}$ au Congo), est utilisée pour le traitement de diverses maladies (Etou-Ossibi et al., 2005) dont, l'hyperthermie et la tension artérielle (Abena et al., 2003). De nombreuses études réalisées indiquent que l'extrait aqueux ou celui de l'huile essentielle de L. multiflora ont des propriétés pharmacologiques (HondiAssah, 2003), pesticides (Oladimeji et al., 2000) et cosmétiques (Kanko et al., 2004). Selon Etou-Ossibi et al. (2016), l'extrait hydroéthanolique de L. multiflora possède des propriétés cardiotoniques. La plante permet de traiter l'hypertension artérielle (Gandonou et al., 2017) et possède des propriétés antifongiques (Tiendrebeogo et al., 2017).

Aujourd'hui, cette espèce est commercialisée et constitue une culture d'exportation en Côte d'Ivoire (Yao-Kouamé et al., 2009). Elle pousse naturellement dans les zones de savanes arborées du centre à Bouaflé,
Tiébissou, Toumodi, Yamoussoukro et dans les savanes herbeuses du Nord à Bondoukou, Bouna, Ferkessédougou, Odienné et Tanda etc.

Cette espèce est sujette aux attaques d'insectes. Selon Kwadjo et al., les fleurs de L. multiflora sont sujettes aux attaques de deux punaises (Hétéroptères) : Tingis sp. (Tingidae) et Poecilocoris latus Dall. (Scutelleridae).

En raison des intérêts économiques croissants, la surexploitation de Lippia multiflora fait courir des menaces de disparition sur celle-ci. Sa sauvegarde et sa domestication sont devenues aujourd'hui une impérieuse nécessité. Selon Yobi et al. (2009), la connaissance précise des caractéristiques morpho-physiologiques et les exigences écologiques d'une espèce végétale constitue la pierre angulaire de tout programme visant à sa conservation, son amélioration et sa domestication. Cependant, l'insuffisance de matériels de plantation, due au faible taux de germination des graines, limite l'extension de la culture de cette espèce en Côte d'Ivoire.

Dans ce contexte, la définition des conditions optimales de germination pour la production de plants à partir de graines, la caractérisation et la conservation semblent indispensables. La présente étude avait pour objectifs la caractérisation (pureté spécifique du lot, diamètre moyen, nombre de graine par unité de masse, taux d'humidité), la germination (Faculté germinative, Vitesse de germination et temps de latence, longévité, dynamique de croissance post-germination) et la conservation des graines de Lippia multiflora Moldenke (Verbenaceae). 


\section{MATERIEL ET METHODES \\ Matériel végétal}

Le matériel végétal est composé de graines de Lippia multiflora Mold. issues de fruits matures, récoltés sur des infrutescences sèches à Yamoussoukro (Figures 1 et 2). Les capsules de la plante ont été cueillies et séchées à la température ambiante. L'extraction des graines a été faite par écrasement des fruits après séchage. Par la suite, une série de tamisage a permis d'éliminer les débris végétaux.

\section{Matériel technique}

Le matériel technique se compose essentiellement d'un sécateur pour la récolte des fruits, de boites de Pétri pour les essais de germination, d'une balance de précision pour les pesées, d'une étuve pour la déshydratation, de papier aluminium pour l'emballage des graines, de plaques alvéolées pour l'élevage des jeunes plantules, de terreau utilisé comme substrat et de piquets en bois pour la délimitation des placettes.

\section{Méthodes \\ Milieu d'étude}

L'étude a été réalisée dans la région du Poro précisément dans le département de Korhogo. Korhogo est une ville située au Nord de la Côte d'Ivoire à $633 \mathrm{~km}$ d'Abidjan (capitale économique et la plus grande ville du pays) et à $356 \mathrm{~km}$ de Yamoussoukro (Capitale politique). Korhogo est le chef-lieu de la région du Poro et du district des savanes et la cinquième métropole de la Côte d'Ivoire. Celle-ci se situe entre les parallèles $8^{\circ} 26$ et $10^{\circ} 27$ de latitude Nord et $5^{\circ} 17$ et $6^{\circ} 19$ de longitude Ouest. La région du Poro est limitée au Nord par la République du Mali, au Sud par la région du Béré, à l'Est par les Régions du Tchologo et du Hambol et à l'Ouest par la Région de la Bagoué. Le département de Korhogo couvre une superficie de $13400 \mathrm{~km}^{2}$, soit 4,2\% du territoire national. (Anonyme, 2013).

\section{Pureté spécifique du lot de semences}

L'analyse de la pureté des semences a été effectuée sur des échantillons de 1 gramme prélevés au hasard dans les lots. Les semences ont été manuellement triées et séparées des impuretés. A l'issue de cette opération, le taux de pureté des semences (TPS) a été évalué selon la formule de l'ISTA (2000) :

TPS $=\frac{\text { poids de semences pures }(g)}{\text { poids total de liechantillon }} \times 100$

\section{Nombre de graines par unité de masse}

Il s'agit de faire le décompte manuel des graines de 5 lots de 1 gramme et de déterminer le nombre moyen de graine par gramme.

\section{Diamètre moyen des graines}

Les graines de Lippia multiflora ont une forme ovale quasi-sphérique. Le diamètre a été évalué sur un lot de 100 graines. Le diamètre moyen est le rapport de la somme des diamètres individuels $\left(D_{i}\right)$ sur le nombre de graine $\left(\mathrm{N}_{\mathrm{g}}\right)$ échantillonné.

\section{Taux d'humidité des graines}

Le taux d'humidité des semences (THS) est la quantité d'eau présente à l'intérieur de celles-ci. Des lots de 1 gramme des graines de Lippia multiflora ont été prélevés puis pesés et mis à l'étuve à $105^{\circ} \mathrm{C}$ pendant $24 \mathrm{~h}$. Ensuite, ils ont été pesés de nouveau pour déterminer le taux d'humidité des semences (THS) selon la formule de l'ISTA (2000) :

$$
\text { THS }(\%)=\frac{\text { poids humide }- \text { poids sec }}{\text { poids humide }} \times 100
$$

\section{Essai de germination}

Il s'agit de déterminer la faculté germinative, la vitesse de germination et les conditions optimales de germination des graines.

Faculté germinative G(\%)

Le comptage des graines germées et les pourcentages cumulés de germination ont été effectués, chaque jour, pendant 30 jours. Une graine est considérée comme ayant germé lorsque la radicule devient visible. Ainsi, le taux de germination G (\%), à un temps donné, est exprimé par le rapport du nombre de graines germées sur le nombre total de graines semées, selon la formule : $G(\%)=\left(\frac{\sum n i}{N}\right) \times 100$ Avec $\mathrm{n}=$ nombre de graines germées et $\mathrm{N}=$ nombre total de graines semées.

Vitesse de germination

La vitesse de germination peut s'exprimer par la durée médiane de germination ou par le temps moyen de germination c'est-àdire le temps au bout duquel on obtient $50 \%$ 
des graines germées (Bayoko, 2020). Dans le cadre de cette étude, le temps maximum de germination (tm), exprimé en jour (j) a été utilisé. C'est le temps au-delà duquel aucune germination de graine n'a été observée. Il est calculé par la formule :

$\operatorname{tm}($ jours $)=\sum\left(\frac{t i \times n i}{N}\right)$

Avec $n_{i}=$ nombre de graines germées au temps $\mathrm{t}_{\mathrm{i}}$ ( $\mathrm{i}$ allant de 1 à 30 jours) et $\mathrm{N}=$ nombre des graines germées à la fin de l'essai.

\section{Durée de viabilité et longévité des graines}

Il s'agit de conserver des lots de graines dans différentes conditions afin de déterminer les meilleures pour les graines de Lippia multiflora après la récolte. Pour ce faire, 2 lots de graines, l'un conservé dans le milieu ambiant et l'autre dans un milieu réfrigéré à la température de $7^{\circ} \mathrm{C}$ ont fait l'objet de tests progressifs de germination.

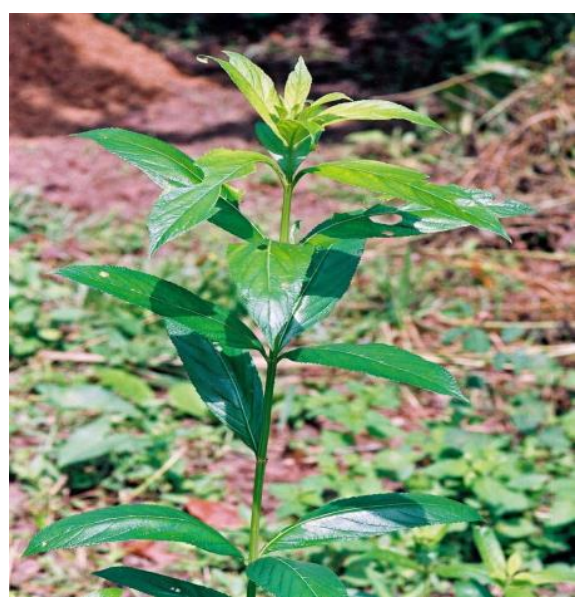

Figure 1 : Jeune plant de Lippia multiflora..

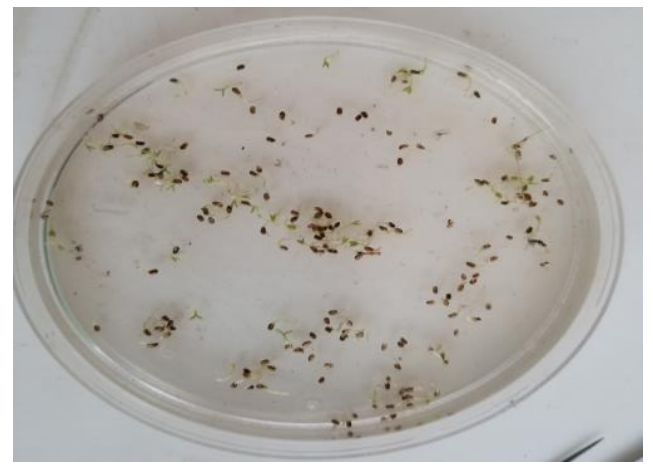

Figure 3 : Graines de Lippia multiflora en germination dans une Boîte de Pétri.

\section{Suivi post-germination des graines}

Il s'agit de faire un suivi postgermination (dynamique de croissance, feuillaison) des plantules de Lippia multiflora dans des conditions au champ dans un dispositif en bloc. Les plantules utilisées proviennent de graines prégermées dans des boites de pétri puis élevés dans des plaques alvéolées (Figures 3 et 4).

\section{Analyses statistiques}

Les données obtenues ont été saisies et analysées à l'aide des logiciels Excel et XLSTAT version 2014. Le pourcentage, la moyenne et l'écart-type des variables ont été calculés. Une ANOVA a été effectuée pour déceler les éventuelles différences significatives entre les traitements. Lorsqu'il y'en a, le test de comparaison des moyennes (Le test de Duncan) a été effectué pour déceler les groupes homogènes.

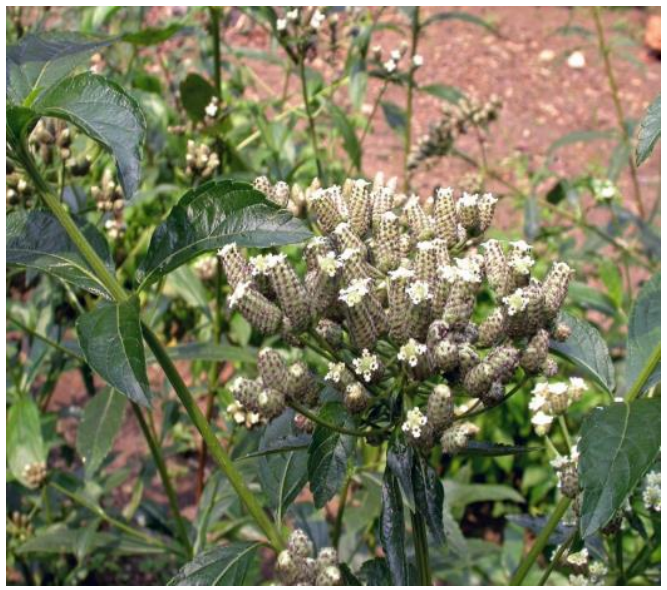

Figure 2 : Infrutescens de Lippia multiflora portant encore des fleurs.

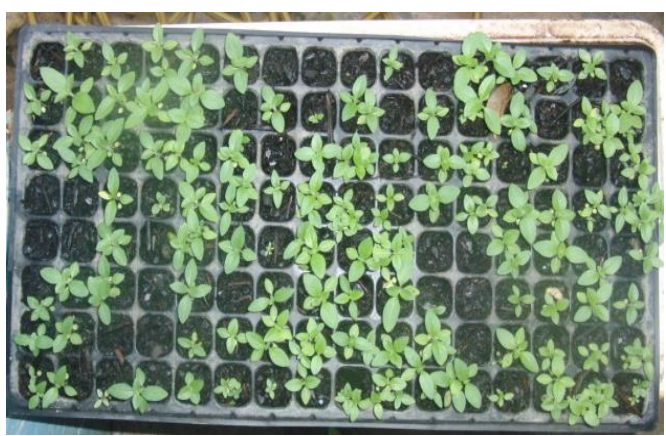

Figure 4 : Plants âgés de deux mois issus des graines dans une plaque alvéolée. 


\section{RESULTATS}

\section{Qualité morphologique du lot et des graines de Lippia multiflora}

L'analyse de la qualité morphologique des graines de Lippia multiflora est consigné dans le Tableau 1. Le test de pureté spécifique des semences effectué sur des échantillons de 1 gramme prélevés au hasard, révèle une pureté spécifique de $70 \pm 10 \%$. Le nombre moyen de graines de Lippia multiflora par gramme est de $4032 \pm 90$. Le diamètre moyen d'une graine est de $0,34 \pm 0,1 \mathrm{~mm}$. La teneur en eau des graines de Lippia multiflora est de $14 \pm 5 \%$.

\section{Qualité physiologique des graines de Lippia multiflora}

Faculté germinative et vitesse de germination des graines

Le taux de germination des graines de Lippia multiflora est $42,25 \%$. Le taux maximal de germination est atteint après 10 jours de semis avec un temps de latence (l'apparition des premières germinations) de 2 jours (Figure 5).

\section{Influence du milieu de conservation sur la} viabilité des graines de Lippia multiflora.

La variation des taux germinatifs en fonction des milieux de conservation et du temps est illustrée par la Figure 6. Le taux de germination initial des graines de Lippia multiflora est de $42.25 \%$. Après un mois conservation dans les milieux ambiants réfrigéré $\left(7^{\circ} \mathrm{C}\right)$, les taux de germination des graines ont été respectivement de 21 et $27.25 \%$ (Tableau 2). L'analyse statistique ne décèle aucune différence significative. Après 2 mois de conservation, les taux de germination ont été respectivement de $13,5 \%$ pour le milieu ambiant et $40,75 \%$ pour le milieu réfrigéré. Statistiquement, la différence entre les deux milieux de conservation est significative $(\mathrm{F}=$ 31,8474; $\mathrm{P}=0,0024)$. Après 3 mois de conservation, des taux germinatifs pour le milieu ambiant et pour le milieu réfrigéré ont été respectivement de $11,75 \%$ et $53 \%$ (Tableau 2). L'analyse statistique montre qu'il y'a une différence significative entre les deux milieux de conservation $(\mathrm{F}=37,4631 ; \mathrm{P}=0,0017)$.

Dynamique de croissance en hauteur et évolution de la feuillaison des plants de Lippia multiflora

Après repiquage en parcelle des plantules de Lippia multiflora, des mensurations sur leur taille montrent un développement régulier de celle-ci dans le temps. Les longueurs moyennes des tiges passent de 2,58 à $8,8 \mathrm{~cm}$ en 3 mois, montrant un accroissement moyen de 6,22 cm (Figure 7). Aucune mortalité des plants n'a été observée. Par ailleurs, le comptage des feuilles des plants indique une évolution croissante de leur nombre, passant de 4 feuilles en moyenne après le repiquage à 22 feuilles après 3 mois (Figure 8).

Tableau 1 : Qualités morphologiques du lot et des graines de Lippia multiflora.

\begin{tabular}{lcccc}
\hline \multicolumn{1}{c}{ Statistiques } & $\begin{array}{c}\text { pureté } \\
\text { spécifique }(\boldsymbol{\%})\end{array}$ & $\begin{array}{c}\text { Nbre de graine } \\
\text { par gramme }\end{array}$ & Diamètre & $\begin{array}{c}\text { humidité relative } \\
(\boldsymbol{\%})\end{array}$ \\
\hline Nb. d'observations & 5 & 5 & 100 & 5 \\
\hline Minimum & 50,00 & 3160,00 & 0,10 & 10,00 \\
\hline Maximum & 90,00 & 5290,00 & 0,60 & 20,00 \\
\hline ler Quartile & 50,00 & 3320,00 & 0,30 & 10,00 \\
\hline Médiane & 70,00 & 3350,00 & 0,30 & 10,00 \\
\hline 3ème Quartile & 90,00 & 5040,00 & 0,40 & 20,00 \\
\hline Moyenne & 70,00 & 4032,00 & 0,3380 & 14,00 \\
\hline Variance (n-1) & 400,00 & 1082770,00 & 0,0117 & 30,00 \\
\hline Ecart-type (n-1) & 10,00 & 1040,5623 & 0,1080 & 5,4772 \\
\hline
\end{tabular}




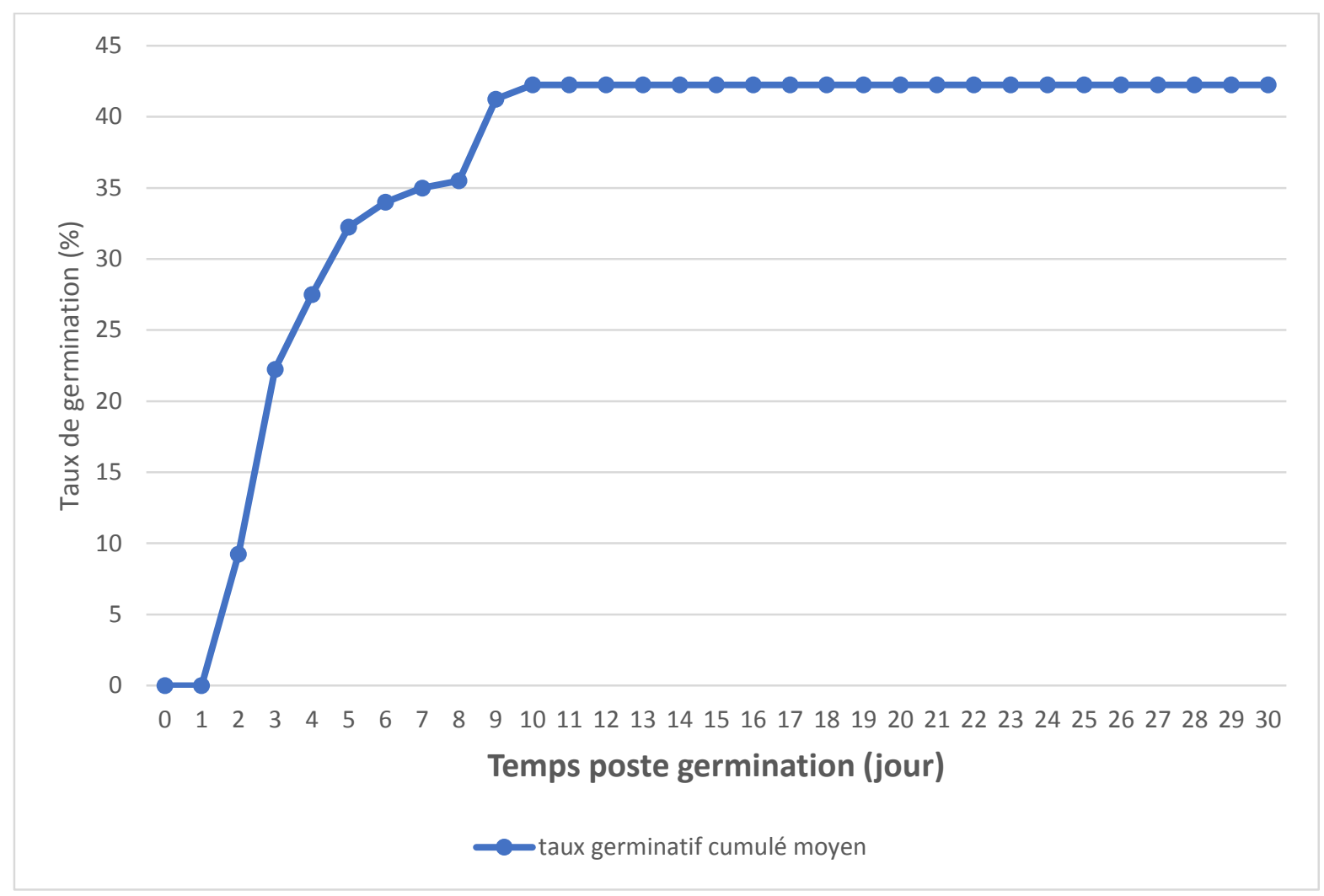

Figure 5 : Evolution du taux de germination des graines Lippia multiflora en fonction du temps.

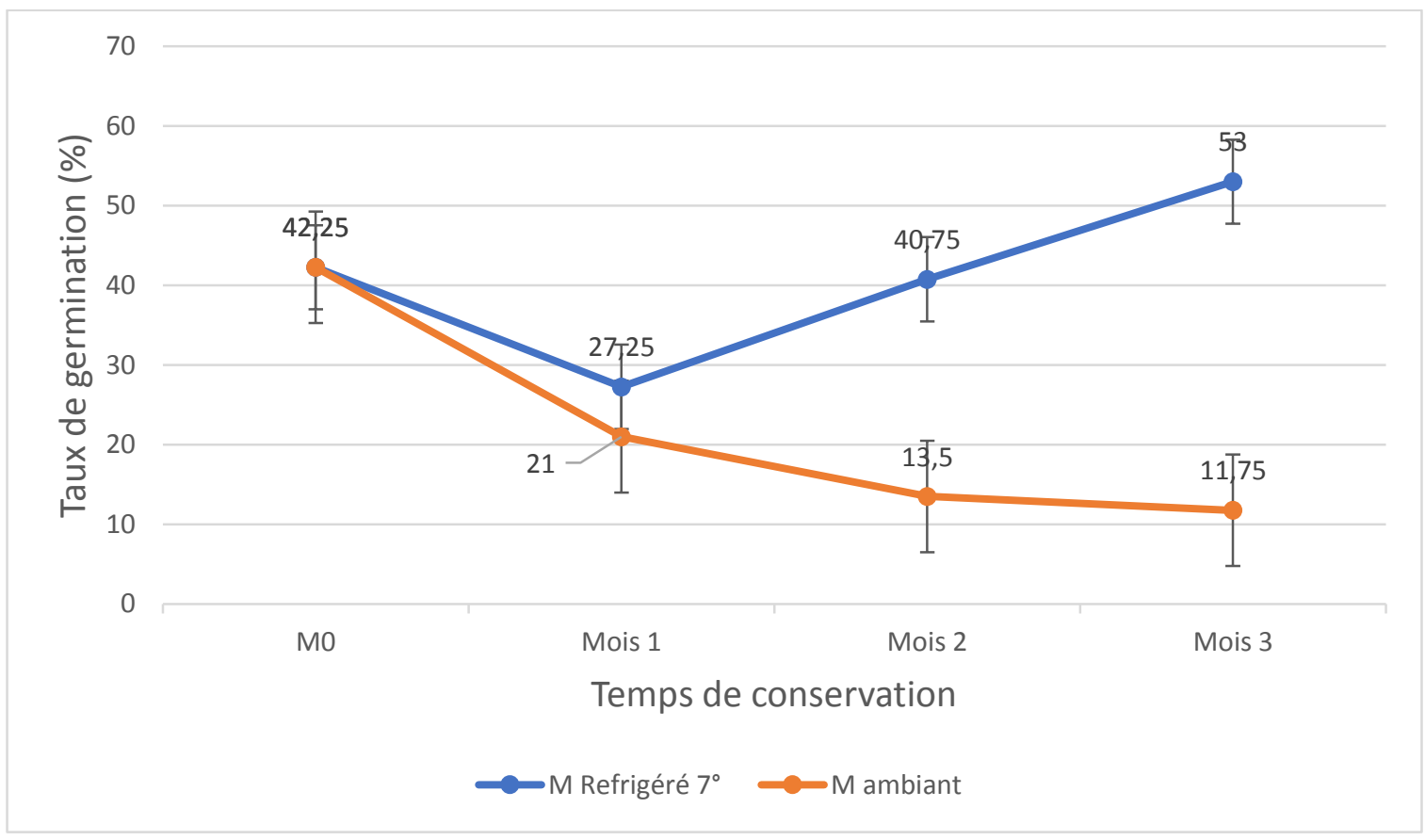

Figure 6: Taux de germination des graines de Lippia multiflora en fonction des milieux de conservation et du temps. 
H. C. K. KONE et al. / Int. J. Biol. Chem. Sci. 14(6): 1988-1998, 2020

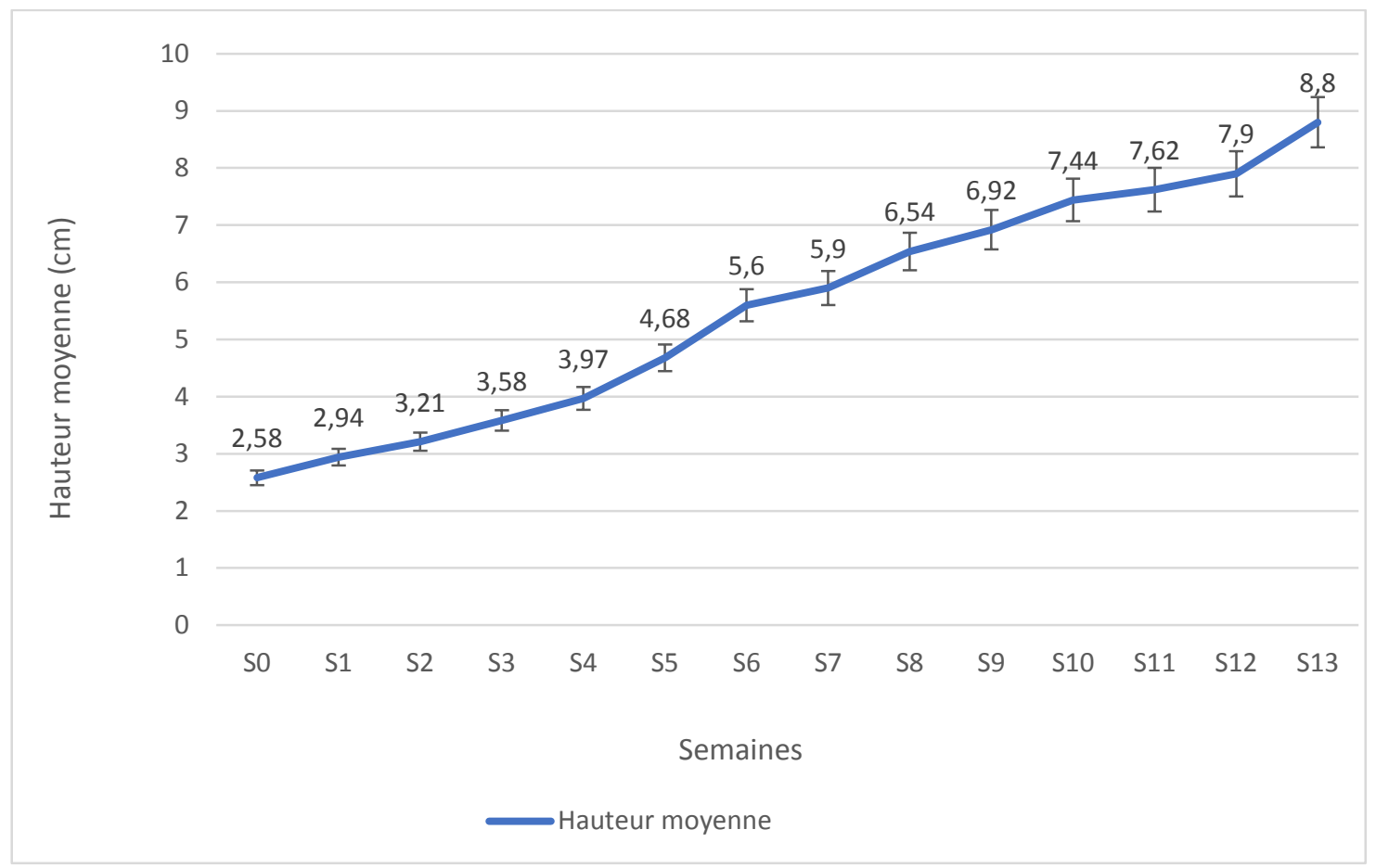

Figure 7 : L'évolution de la croissance en hauteur des plants de Lippia multiflora en fonction du temps.

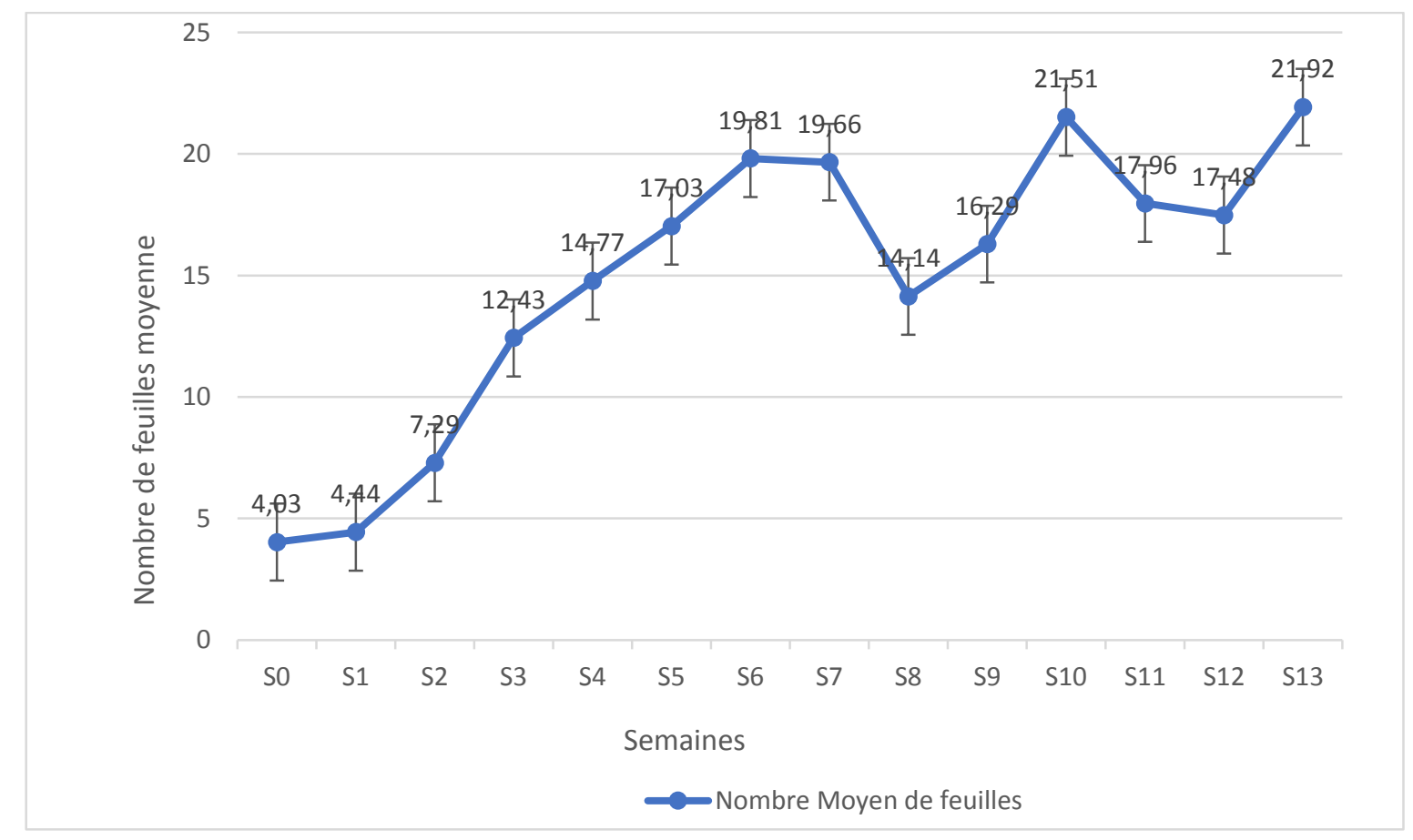

Figure 8 : Evolution de la feuillaison des plants de Lippia multiflora en fonction du temps (semaines). 
Tableau 2 : Analyse statistique des taux germinatifs en fonction des milieux de conservation et du temps de conservation des graines de L. multiflora.

\begin{tabular}{lllll}
\hline Conservation & Modalité & Moyenne estimée & $\mathbf{F}$ & Pr $>\mathbf{F}$ \\
\hline 1 mois & Trefrigéré $7^{\circ}$ & $27,25 \mathrm{a}$ & 1,6347 & 0,2483 \\
\hline & T ambiante & $21 \mathrm{a}$ & & \\
\hline 2 mois & Trefrigéré $7^{\circ}$ & $40,75 \mathrm{a}$ & 45,0607 & 0,0005 \\
\hline & T ambiante & $13,5 \mathrm{~b}$ & & \\
\hline 3 mois & T refrigéré $7^{\circ}$ & $53 \mathrm{a}$ & 49,9541 & 0,0004 \\
\hline & T ambiante & $11,75 \mathrm{~b}$ & & \\
\hline
\end{tabular}

Les moyennes affectées d'une même lettre, dans une même colonne, ne sont pas significativement différentes au seuil $\alpha<0,05$, selon la méthode de Duncan.

\section{DISCUSSION}

Le lot de graines de Lippia multiflora se caractérise par une pureté spécifique de l'ordre de $70 \%$. Les débris contenus dans les graines $(30 \%)$ ont quasiment la même taille et le même poids que les graines, ce qui rend difficile la purification. Les graines sont de très petite taille voire microscopique. Le nombre moyen de graines par gramme est de 4032 avec un diamètre moyen de l'ordre de 0,34 millimètre. Selon Bayoko (2020), les graines dont la teneur en eau à maturité varie entre 10 et $15 \%$ sont qualifiées de sèches. Celles-ci contiennent donc peu d'eau avec des réactions enzymatiques ralenties ce qui permet de bien les conserver. Lippia multiflora dont la teneur en eau est de l'ordre de $14 \%$ peut être rangée dans cette catégorie de graines sèches.

Les tests de germination effectués sur le lot de semences montrent que le taux de germination des graines de Lippia multiflora est $42,25 \%$, le temps moyen que mettent les radicules à perforer les téguments est de 2 jours après imbibition. Le taux maximal de germination des graines est atteint 10 jours après la mise en germination. Ces résultats diffèrent de ceux de Soumahoro et al. (2014), qui ont trouvé un taux de germination de $59.01 \%$ pour des tests de germination réalisés sur du papier filtre, avec des graines imbibées directement dans l'eau pendant $24 \mathrm{~h}$.

La durée de viabilité des graines au cours du temps est un paramètre très important pour la conservation des ressources phytogénétiques. Cette viabilité chute plus ou moins rapidement en fonction des espèces. Le record en la matière semble être détenu par Lotus Nelumbo-nucifera avec une longévité de l'ordre de 800 à 1000 ans.

La comparaison des taux de germinations des graines dans 2 milieux de conservation révèle que ces graines se conservent mieux à basse température. Les graines de Lippia multiflora ne présentent pas de dormance, celles-ci germent dès que les conditions sont réunies. Selon Bayoko (2020), la germination est un phénomène qui est influencé par des facteurs, aussi bien, internes, qu'externes, notamment, la lumière, la présence de l'eau, l'oxygène, la température, la perméabilité des enveloppes de la graine, le génome, l'âge de la graine, la nature du substrat, etc. La conservation des graines dans le milieu réfrigéré conserve et améliore la capacité germinative des graines. Ainsi, le milieu réfrigéré influence positivement la faculté germinative des graines tandis que dans le milieu ambiant, le taux de germination des graines baisse rapidement. Il a été rapporté par Oladiran et Ogunbiade (2000) que la température élevée, couplée à la faible quantité d'eau produite au cours de la respiration, sont susceptibles d'élever la température ambiante autour d'un lot de semences, et, par voie de conséquence, affecter, de façon significative, leur viabilité lorsque la graine est conservée pendant longtemps. C'est ce qui pourrait expliquer le faible taux de germination des 
graines de Lippia multiflora seulement après 3 mois de conservation dans le milieu ambiant. Dans le cas des graines, avant de conduire, de façon irréparable, à la mort cellulaire, l'accumulation d'une succession de dégâts des structures biologiques au cours du stockage affecte progressivement la vigueur germinative. Ces phénomènes de détérioration se produisent, même dans des conditions idéales de conservation. La durée de vie d'une graine est déterminée par son potentiel génétique et physiologique de conservation et par les conditions environnementales qu'elle rencontre lors de son stockage. Des travaux très récents démontrent qu'il existe des inégalités et une très grande hétérogénéité entre les graines des différentes espèces de plantes par rapport à leur capacité à supporter la durée de conservation (Walters et al., 2005). Au-delà des conditions de stockage, la capacité de conservation des graines dépend de leurs propriétés intrinsèques à protéger l'embryon par les caractéristiques physiques et chimiques de l'enveloppe (Debeaujon et al., 2000; Sattler et al., 2004), mais aussi du maintien d'une activité métabolique réduite. Le maintien de la vigueur germinative, au cours du stockage et du vieillissement des graines est conditionné par le maintien de la capacité de l'embryon à éliminer des composés toxiques (formes réactives de l'oxygène), et à réparer ou à renouveler les constituants cellulaires altérés au cours du temps (Buitink et al., 2000).

Avec une hauteur moyenne de $2,58 \mathrm{~cm}$ au repiquage les plantes gagnent en hauteur pour atteindre une moyenne de $8,8 \mathrm{~cm}$ tandis que le nombre de feuille augmente au fur et à mesure ; passant de 4,03 feuilles en moyenne, après le repiquage à 21,9 feuilles après trois mois. Ces résultats diffèrent de ceux de Alui et al., (2011) qui ont obtenu, après 60 jours de mensuration, un accroissement en hauteur de $7,44 \mathrm{~cm}$. Cela pourrait s'expliquer par la zone phytogéographique et la période de mise en place de l'essai. En effet, Alui et al., 2011 ont travaillé en basse Côte d'Ivoire où la pluviométrie est plus élevée.

\section{Conclusion}

L'étude des paramètres morphophysiologiques montre que les graines de Lippia multiflora se caractérisent par un diamètre moyen de 0,34 ; une pureté spécifique de $70 \%$, une teneur en eau de $14 \%$. Le taux de germination des graines de Lippia multiflora est $42,25 \%$. Après différents tests de germination pour identifier les meilleures conditions de conservation des graines, il en ressort que celles-ci se conservent mieux en milieu réfrigéré. Le suivi post-germination des plantules au champ montre une évolution lente mais régulière de la hauteur des plants. Durant ce temps d'essai au champ, aucune mortalité n'a été observée malgré les conditions drastiques de sécheresse dans la région d'étude. A l'issu de cette étude, nous pouvons conclure que Lippia multiflora est une plante fort résiliente qui peut s'adapter aux conditions pédoclimatiques du Nord de la Côte d'Ivoire. L'agro-biodiversité étant une des solutions pour la sécurité alimentaire, face à la menace des changements climatiques, il est impératif de pérenniser cette plante, par la promotion et la valorisation de sa culture, mais aussi celles des savoirs locaux. Par la suite, une comparaison de la qualité des huiles essentielles extraites des feuilles en fonction des zones phytogéographiques devrait permettre d'identifier les meilleures zones de production et de culture de Lippia multiflora en Côte d'Ivoire.

\section{CONFLIT D'INTERETS}

Les auteurs attestent qu'il n'existe aucun conflit d'intérêts entre eux ou autre partie au sujet de cet article.

\section{CONTRIBUTIONS DES AUTEURS}

KKHC a rédigé le protocole d'expérimentation, contribué à la mise en place de l'essai, à la vérification et à l'exploitation des données (analyses statistiques) ainsi qu'à la rédaction de la première version du manuscrit. ATN et CLF ont veillé à la conformité des résultats et contribué à la rédaction du manuscrit. BF a participé à la mise en place de l'essai, au suivi et à la récolte des données. 


\section{REMERCIEMENTS}

Nos remerciements vont à l'endroit de Docteur N'GUESSAN Aya Carine, enseignant-chercheur à l'Université Péléfero GON COULIBALY qui nous a mis gracieusement à disposition le matériel de laboratoire.

\section{REFERENCES}

Abena AA, Diatewaa M, Gakossoa G, Gbeassorc M, Hondi-Assaha TH, Ouamba JM. 2003. Analgesic, antipyretic and anti-inflammatory effects of essential oil of Lippia multiflora. Fitoterapia, 74: 231-236.

DOI : https://www.ncbi.nlm.nih.gov/pubmed/1 2727486

Ambé GA. 2001. Les fruits sauvages comestibles des savanes guinéennes de Côte d'Ivoire : état de la connaissance par une population locale, les Malinkés. Biotechnol. Agron. Soc. Environ., 5 (1) : 43-58. DOI : https://www.docdeveloppement- durable.org/file/ArbresFruitiers/FICHES_ARBRES/tamarinier/ Les\%20fruits\%20sauvages\%20comestibl es $\% 20$ des $\% 20$ savanes $\% 20 \% 20 \mathrm{de} \% 20 \mathrm{Co}$ te $\% 20 \mathrm{~d} \% 20$ ivoire.pdf

Alui KA, Yao-Kouamé A, Ballo KC, Kouadio KP, N'guessan KA, Nangah KY. 2011. Comportement de deux morphotypes de Lippia multiflora (Verbenaceae) sur Ferralsols de la région de Yamoussoukro, Côte d'Ivoire. Journal of Applied Biosciences, 38: 2592-2601. DOI: http://www.m.elewa.org/JABS/2011/38/ 10.pdf

Anonyme. 2013. Article Korhogo, Conseil Régional de Korhogo. www.regionduporo.org.

Bayoko F. 2020. Caractérisation morphophysiologique et conservation des graines de Lippia multiflora Moldenke. Mémoire de fin d'étude pour l'obtention du diplôme de Master en Protection des Végétaux et de l'Environnement, UFR des Sciences Biologiques, Université Péléfero GON COULIBALY, 67 p.

Buitink J, Leprince $\mathrm{O}$, Hernrninga MA, Hoekstra FA. 2000. Molecular mobility in the cytoplasrn: an approach to describe and predict lifespan of dry gerrnplasm. Proc Natl Acad Sei USA, 97: 2385-2390. http://europepmc.org/article/PMC/15810 Debeaujon I, Léon-Kloosterziel KM, Koornneef M. 2000. Influence of the testa on seed dormancy, germination, and longevity in Arabidopsis. Plant Physiol, 1 (22): 403-413. DOI: https://www.ncbi.nlm.nih.gov/pubmed/1 0677433

Etou-Ossibi W, Nzonzi J, Mombouli JV, Nsondé-Ntandou GE, Ouamba JM, Abena AA. 2005. Screening chimique et effet de l'extrait aqueux de Lippia multiflora Moldenke sur le cœur isolé de crapaud. Phytothérapie, 3(5) : 193-198. http://archives.uvci.edu.ci:52003/data/U NA/import_sauvegarde_08032018_una/ MEMOIRE_636936051552325930.pdf

Etou-Ossibi AW, Elion-Itou RDG, Morabandza CJ, Nsonde-Ntandou GF, Nzonzi J, Ouamba JM, Abena AA. 2016. Effets de l'extrait hydroéthanolique de Lippia multiflora Moldenke sur le cœur isolé de crapaud. Int. J. Biol. Chem. Sci., 10(6): 2617-2636. DOI: http://dx.doi.org/10.4314/ijbcs.v10i6.17

Gandonou DC, Ahissou H, Tokoudagba J-M, Dansou C. 2017. Ethnobotanical, phytochemical and toxicity analysis of a Beninese antihypertensive plant: Lippia multiflora. Int. J. Biol. Chem. Sci., 11(4): 1816-1828.

DOI: http://dx.doi.org/10.4314/ijbcs.v11i4.31

Hondi-Assah C, Abena AA, Kokolo J, Badi AC, Diatewa M. 2003. Effets hépatoprotecteurs de Lippia multiflora et d'un phytomédicament congolais. Phytothérapie, 1(5): 135-140. DOI: $10.1007 / \mathrm{s} 10298-016-1025-8$

ISTA. 2000. International Rules for Seed Testing International Seed Testing Association, Edition 2000. Bassersdorf, Suisse: $204 \mathrm{p}$.

Kanko C, Sawaliho BEH, Kone S, Koukoua G, N'Guessan YT. 2004. Etude des propriétés physico-chimiques des huiles essentielles de Lippia multiflora, Cymbopogon citratus, Cymbopogon 
nardus, Cymbopogon giganteus. Comptes Rendus Chimie, 7: 1039-1042. DOI : https://doi.org/10.1016/j.crci.2003.12.03 0

Kwadjo EK, Kra DK, Doumbia M. 2011. Données préliminaires sur l'entomofaune de Lippia multiflora Mold. (verbenaceae) en Côte d'Ivoire. Agronomie Africaine, 23 (1) : 53 - 59.

Oladimeji FA, Orafidiya OO, Ogunniyi TAB, Adewunmi TA. 2000. Pediculocidal and scabicidal properties of Lippia multiflora (Verbenaceae) essential oil. Journal of Ethnopharmacology, 72 (1-2): 305-311. DOI: https://doi.org/10.1016/S03788741(00)00229-4

Oladiran JA, Ogunbiade SA. 2000. Germination and seedling development from pepper (Capsicum annuum L.) seeds following storage in different packaging materials. Seed Science and Technology. 28: 413-419. DOI: https://doi.org/10.1556/AAgr.50.2002.2. 6

Sattler SE, Gilliland LU, MagallanesLundback M, Pellard M, DellaPenna O. 2004. Vitamin E 1s Essential for Seed Longevity and for Preventing Lipid Peroxidation during Germination. Plant Cell, 16: 1419-1432. DOI: $10.1105 /$ tpc. 021360
Soumahoro AB, Koné T, Koné M, Konate $\mathrm{S}$, Kouadio JY, Zouzou M. 2014. Protocole efficace de germination des graines de Lippia multiflora. Agronomie Africaine, $\quad 26$ (2): 137 - 145. DOI : https://www.ajol.info/index.php/aga/artic le/view/107199

Tiendrebeogo A, Ouedraogo I, Bonzi S, Kassankogno AI. 2017. Etude de l'activité antifongique d'extraits de Cymbopogon citratus (DC.) Stap, Eclipta alba L., Lippia multiflora M. et Agave sisalana P. Int. J. Biol. Chem. Sci., 11(3): 1202-1211.

DOI: https://dx.doi.org/10.4314/ijbcs.v11i3.22

Walters CT, Wheeler LJ, Grotenhuis JA. 2005. Longevity of seeds stored in a genebank: Species characteristics. Seed Sei Res, 15 : 1-20. DOI: $10.1093 /$ conphys/coy033

Yao-Kouamé A, Allou K. 2008. Propriétés du sol et domestication de Lippia multiflora (Verbenaceae) en Côte d'Ivoire. Agronomie Africaine, 20(1): 97-107. DOI: 10.4314/aga.v20i1.1739

Yobi A, Henchi B, Mohamed N, Jendoubi R. 2009. Système de reproduction et variabilité morpho-phénologique chez Allium roseum L. Plant Genetic Ressources Newsletter, 127: 29 - 34. https://www.bioversityinternational.org/f ileadmin/PGR/article-issue_153-art_5lang_en.html 